\title{
Polyamine Transport and Role of potE in Response to Osmotic Stress in Escherichia coli
}

\author{
DIRK SCHILLER, ${ }^{1}$ DANIELA KRUSE, ${ }^{1}$ HELMUT KNEIFEL, ${ }^{2}$ REINHARD KRÄMER,${ }^{1}$ \\ AND ANDREAS BURKOVSKI ${ }^{1 *}$ \\ Institut für Biochemie, Universität Köln, D-50674 Cologne, ${ }^{1}$ and Forschungszentrum \\ Jülich, ICG6, D-52425 Jülich, ${ }^{2}$ Germany
}

Received 7 June 2000/Accepted 15 August 2000

\begin{abstract}
When transport of polyamines in Escherichia coli was examined, putrescine excretion was observed under two different physiological conditions: (i) strictly correlated to growth and (ii) following a hyperosmotic shock. Spermidine was not excreted. Characterization of a deletion mutant showed that PotE is not involved in these transport processes.
\end{abstract}

The response of Escherichia coli challenged by hyperosmotic stress can be separated into three phases $(2,10)$. (i) Immediately after hyperosmotic shock the cells shrink due to massive water efflux. (ii) Subsequently, this process is counteracted by an increase in internal solute concentration due to uptake of potassium ions and synthesis of glutamate. Additionally, compatible solutes like glycine betaine, proline, and trehalose are accumulated. (iii) After establishing stable osmotic conditions, DNA replication, protein synthesis, and growth are resumed. The fast influx of potassium ions increases not only osmolarity, but also the number of positive charges within the cell. Although this is partially balanced by synthesis of glutamate, polyamine efflux may occur to compensate for the charges transported into the cell due to potassium uptake (7). Since we are interested in osmoregulation and related transport processes, we reexamined polyamine transport in E. coli. To circumvent possible complications of previous studies, which required preloading of cells with labeled polyamines, preparation of insideout vesicles, or overproduction of transport proteins (5), here we analyzed polyamines directly via reversed-phase high-pressure liquid chromatography (HPLC). Thus, we were able to examine polyamine fluxes under physiological conditions.

Growth-dependent polyamine excretion. While polyamine excretion by proliferating eukaryotic cells is widely accepted, it is still under dispute in prokaryotes. In contrast to Tabor and Tabor (8), who deny polyamine excretion by bacteria growing in minimal medium, Kashiwagi et al. (5) exactly propose this flux. We investigated polyamine transport in E. coli wild-type AN387 (9) during growth in M9 minimal medium. Putrescine was excreted in amounts strictly correlated to the cell mass (Fig. 1A), while release of other polyamines like spermidine and cadaverine was not detected. The reason for putrescine efflux during growth is unclear.

Excretion of polyamines after hyperosmotic shock. To examine polyamine transport upon hyperosmotic stress, cells

\footnotetext{
* Corresponding author. Mailing address: Institut für Biochemie, Universität Köln, Zülpicher-Str. 47, D-50674 Cologne, Germany. Phone: 49-221-470-6472. Fax: 49-221-470-5091. E-mail: a.burkovski @uni-koeln.de.
}

grown in M9 minimal medium were washed with warmed medium to remove putrescine excreted during growth, resuspended in fresh medium, and subsequently subjected to a hyperosmotic shock by addition of $0.5 \mathrm{M} \mathrm{NaCl}$. As a consequence, strain AN387 started immediately to excrete putrescine, elevating its concentration in the supernatant from 0 to $25 \mu \mathrm{M}$ within $30 \mathrm{~min}$, while in an untreated control culture, an increase of only $8 \mu \mathrm{M}$ due to growth-dependent excretion was observed (Fig. 1B).

Polyamine transport and accumulation of glutamate and potassium. To study if the hyperosmotic shift-dependent polyamine excretion is necessary to maintain the charge balance of the cell, internal polyamine, glutamate, and potassium concentrations were determined. For this purpose, cells were grown in K5 medium (3), washed and resuspended in warmed K5 medium, and subjected to an osmotic upshift. Following hyperosmotic shock, strain AN387 started immediately to excrete putrescine, and in the course of the experiment, internal putrescine decreased with a rate of approximately $1 \mathrm{nmol}$ per mg (dry weight) per min from 45 to $5 \mathrm{nmol}$ per mg (dry weight), while external putrescine concentrations rose from 0 to $53 \mu \mathrm{M}$ (Fig. 2A). It was calculated that the increase in external putrescine exactly corresponded to its decrease in the cell. Changes in spermidine concentrations were not detected.

When the culture was diluted with an equal volume of distilled water to study the effect of hypoosmotic shock on polyamine transport as well, (i) polyamines were not released via mechanosensitive channels activated under these conditions (7) (Fig. 2B) and (ii) a small increase in internal putrescine with a rate of $0.3 \mathrm{nmol}$ per $\mathrm{mg}$ (dry weight) per min was observed.

Following hyperosmotic shock, $\mathrm{K}^{+}$accumulated with a rate of $22 \mathrm{nmol}$ per mg (dry weight) per min from 400 to $1,540 \mathrm{nmol}$ per mg (dry weight). At the same time, glutamate was synthesized with a rate of 14 nmol per mg (dry weight) per min, reaching up to $800 \mathrm{nmol}$ per mg (dry weight) 40 min after the upshift (Fig. 2B). Upon hypoosmotic shock, both glutamate and $\mathrm{K}^{+}$were released via mechanosensitive channels, as described previously (7).

In summary, glutamate synthesis alone cannot fully compensate for potassium uptake in response to hyperosmotic shock. Only around $700 \mathrm{nmol}$ per $\mathrm{mg}$ (dry weight) of negative charges were accumulated in response to the influx of 1,100 nmol per 


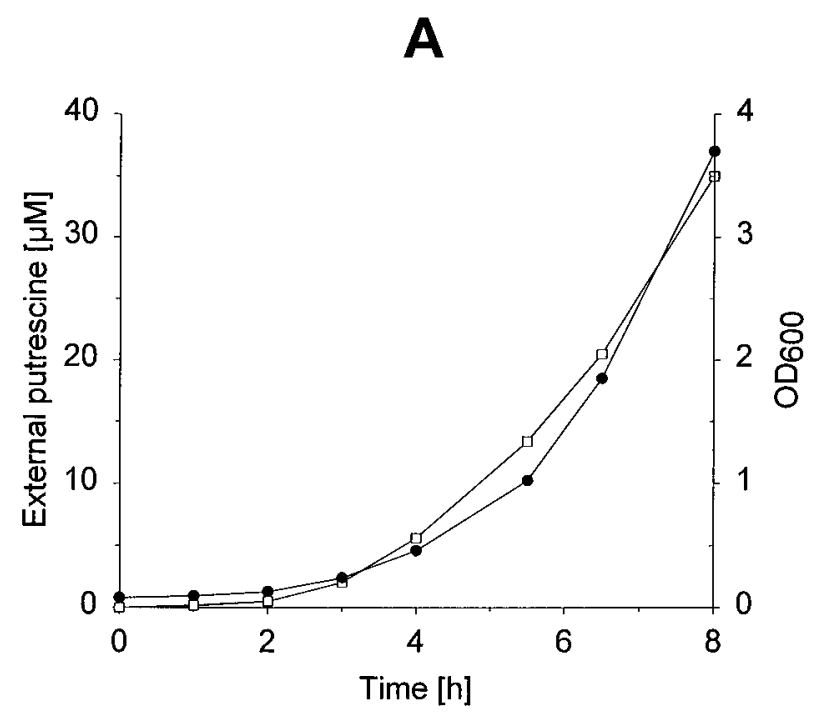

B

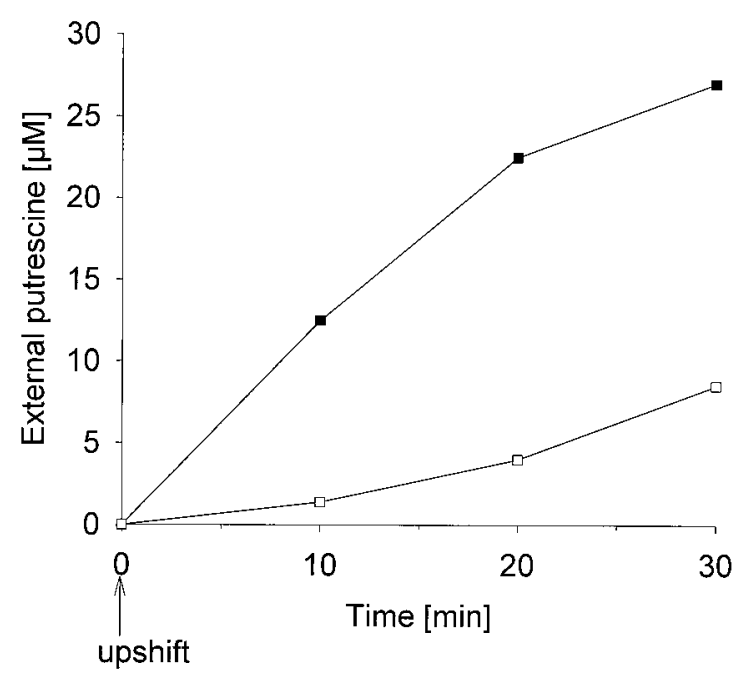

FIG. 1. Excretion of putrescine by strain AN387 grown in M9 medium. (A) Growth-dependent increase of external putrescine (solid circles, $\mathrm{OD}_{600}$; open squares, external putrescine). (B) Putrescine concentrations depending on hyperosmotic shock (solid squares, external putrescine after osmotic upshift; open squares, untreated control). $\mathrm{NaCl}(0.5 \mathrm{M})$ was added at time zero (indicated by an arrow).

mg (dry weight) of positive charges. Putrescine transport contributed with an efflux of $100 \mathrm{nmol}$ per mg (dry weight) of positive charges to the cellular charge balance. Spermidine has other functions than a role in osmoadaptation, as proposed previously $(2,6)$.

Role of potE. Polyamines are highly hydrophilic, and passive diffusion through the cytoplasmic membrane is negligible. Two ABC transporters were described for the uptake of putrescine and spermidine besides an ornithine/putrescine antiporter, encoded by potE, which was considered for putrescine uptake and excretion (4). Since we were interested to identify the putrescine efflux system, we investigated the role of the potE gene. Transcription of potE was described to be acid inducible (4). Therefore, we first tested the expression of pot $E$ in different media by reverse transcription

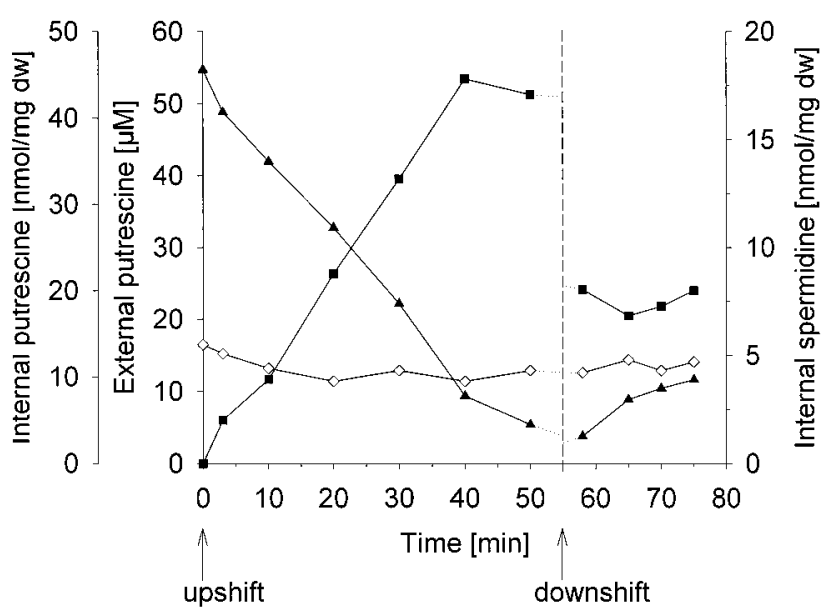

B

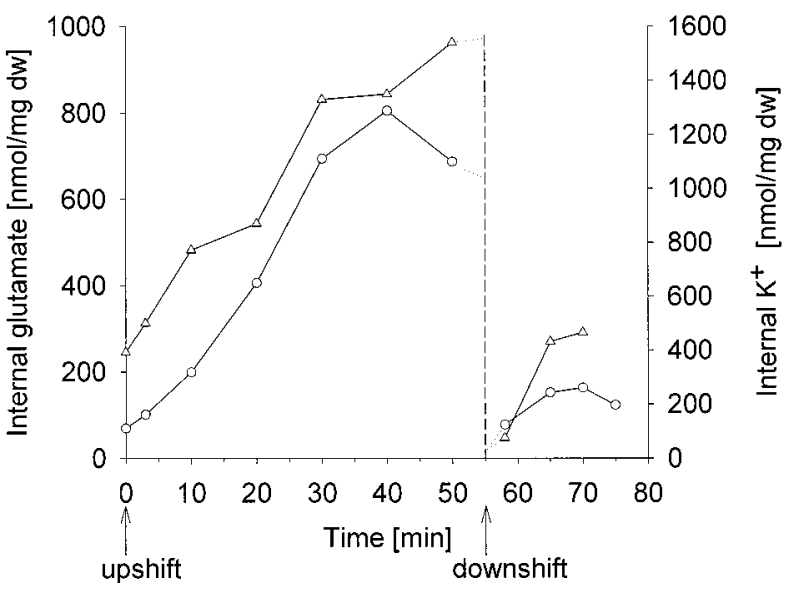

FIG. 2. Solute fluxes in strain AN387 grown in K5 medium. The time of addition of $0.5 \mathrm{M} \mathrm{NaCl}$ (upshift) and an equal volume of distilled water (downshift) is indicated by arrows. (A) Polyamine concentrations depending on osmotic stress (solid squares, external putrescine; solid triangles, internal putrescine; open diamonds, internal spermidine). dw, dry weight. (B) Internal glutamate and potassium concentrations depending on osmotic stress (open circles, glutamate; open triangles, potassium)

(RT)-PCR. A potE transcript was detected when RNA was used as template prepared from AN387 cells grown in M9, $\mathrm{K} 5$, or K5 medium adjusted to $\mathrm{pH} 5.5$ and supplemented with $0.5 \mathrm{mM}$ ornithine (Fig. 3). The absence of contaminating chromosomal DNA was verified in a control PCR without RT (data not shown). The increased amount of RT-PCR product found when RNA prepared from cells grown at $\mathrm{pH}$ 5.5 in the presence of ornithine was used as a template indicated that potE expression is enhanced under these growth conditions.

To avoid possible artifacts caused by overproduction of a membrane protein, an unmarked deletion of the complete pot $E$ gene was introduced in the $E$. coli chromosome using the method described by Blomfield et al. (1). The deletion was verified by PCR (data not shown). When the resulting strain, $\Delta$ potE, was characterized with respect to polyamine transport, no effects on putrescine excretion during growth 


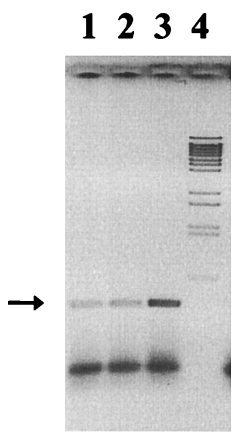

FIG. 3. Transcription of potE. Products of RT-PCR using total RNA as the template prepared from cells grown in M9, K5, and $\mathrm{K} 5$ medium adjusted to $\mathrm{pH}$ 5.5 (lanes 1 to 3, respectively), and BstEII-digested $\lambda$ DNA (lane 4). The 0.6-kb potE gene product is indicated by an arrow.

(data not shown) and on putrescine and spermidine transport upon hyperosmotic shock were observed. Upon hyperosmotic stress, internal putrescine concentrations decreased and external putrescine concentrations increased, while intracellular spermidine stayed constant (Fig. 4). Also, putrescine reaccumulation after hypoosmotic shock was not impaired. Accumulation of glutamate and $\mathrm{K}^{+}$was tested as a control. Both total accumulation and rate of synthesis and uptake were identical to those found for the parental strain AN387 (data not shown). Moreover, no differences between wild-type and $\Delta$ potE strains were obtained when both were grown in K5 medium adjusted to $\mathrm{pH} 5.5$ and when $0.5 \mathrm{mM}$ ornithine was added to activate the PotE antiport mechanism (data not shown).

Obviously, PotE is not involved in growth- or osmodependent putrescine transport in defined minimal medium. In the wild type, without overproduction of PotE, putrescine excretion was not dependent on externally added ornithine, i.e., an ornithine/putrescine antiport mode can be excluded. Since putrescine excretion previously measured for PotE (5) was determined in the antiport mode with externally added ornithine and after overproduction of this carrier protein, it can be concluded that a putrescine exporter not recognized until now is present in E. coli.

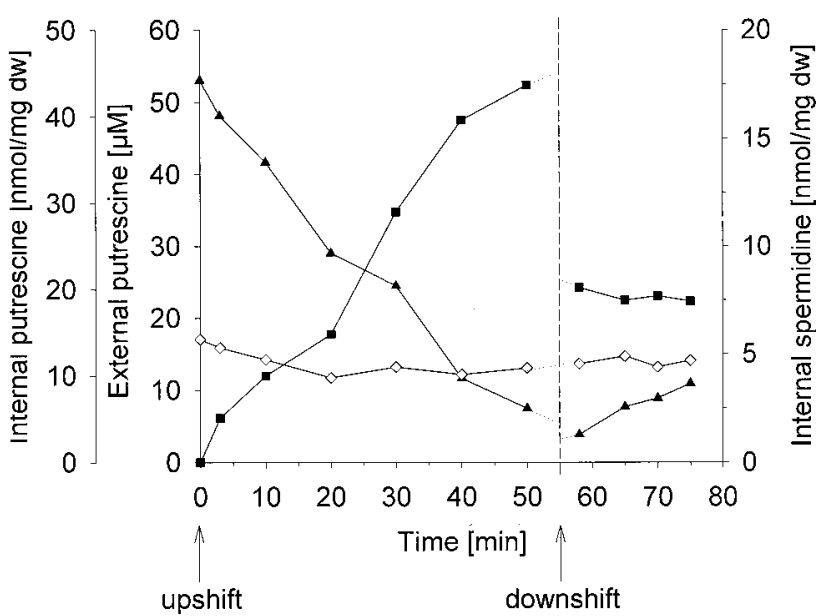

FIG. 4. Solute fluxes in strain $\Delta p o t E$ grown in K5 medium. For explanations, see the legend to Fig. 2.

\section{REFERENCES}

1. Blomfield, I. C., V. Vaughn, R. F. Rest, and B. I. Eisenstein. 1991. Allelic exchange in Escherichia coli using the Bacillus subtilis sacB gene and a temperature-sensitive pSC101 replicon. Mol. Microbiol. 5:1447-1457.

2. Csonka, L. N., and W. Epstein. 1996. Osmoregulation, p. 1210-1224. In F. C. Neidardt et al. (ed.) Escherichia coli and Salmonella typhimurium: cellular and molecular biology. American Society for Microbiology, Washington, D.C.

3. Epstein, W., and B. S. Kim. 1971. Potassium loci in Escherichia coli. J. Bacteriol. 108:639-644.

4. Igarashi, K., and K. Kashiwagi. 1999. Polyamine transport in bacteria and yeast. Biochem. J. 344:633-642.

5. Kashiwagi, K., S. Shibuya, H. Tomitori, A. Kuraishi, and K. Igarashi. 1992. Excretion of putrescine by the putrescine-ornithine antiporter encoded by the potE gene of Escherichia coli. Proc. Natl. Acad. Sci. USA 89:4529-4533.

6. Munro, G., K. Hercules, J. Morgan, and W. Sauerbier. 1972. Dependence of the putrescine content of Escherichia coli on the osmotic strength of the medium. J. Biol. Chem. 247:1272-1280.

7. Schleyer, M., R. Schmid, and E. P. Bakker. 1993. Transient, specific and extremely rapid release of osmolytes from growing cells of Escherichia coli K-12 exposed to hypoosmotic shock. Arch. Microbiol. 160:424-431.

8. Tabor, C. W., and H. Tabor. 1985. Polyamines in microorganisms. Microbiol. Rev. 49:81-99.

9. Wallace, B. J., and I. G. Young. 1977. Role of quinones in electron transport to oxygen and nitrate in Escherichia coli. Biochim. Biophys. Acta 461:84-100.

10. Wood, J. 1999. Osmosensing by bacteria: signals and membrane-based sensors. Microbiol. Mol. Biol. Rev. 63:230-262. 